\title{
Nanotube-based source of charges for experiments with solid helium at low temperatures
}

D. N. Borisenko

Institute of Solid State Physics RAS, Chernogolovka, Moscow Region 142432, Russia

P. M. Walmsley and A. I. Golov

The University of Manchester, Oxford Road, Manchester M13 9PL, United Kingdom

N. N. Kolesnikov, Yu. V. Kotov, A. A. Levchenko, ${ }^{\text {a) }}$ and L. P. Mezhov-Deglin

Institute of Solid State Physics RAS, Chernogolovka, Moscow Region 142432, Russia

\section{J. Fear}

The University of Manchester, Oxford Road, Manchester M13 9PL, United Kingdom

(Submitted February 2, 2015)

Fiz. Nizk. Temp. 41, 729-732 (July 2015)

\begin{abstract}
Methods of preparation of the field-emission sources of charges from carbon nanotubes suitable for study of injected charges in solid helium at low temperatures $T<1 \mathrm{~K}$ are presented. The sources have been prepared by arc discharge deposition of nanotubes onto a flat copper substrate or by mechanical rubbing of nanotubes into porous metal surface. The test study of the voltage-current characteristics of a diode cell with the nanotube source in superfluid He II have shown that at voltages above $120 \mathrm{~V}$ one can observe a relatively large current $I \geq 10^{-13}$ A of negative charges in liquid helium. The field and temperature dependences of positive and negative currents in solid ${ }^{4} \mathrm{He}$ were studied in samples grown by the blocked capillary technique. Usage of the nanotube based source of injected charges had permitted us for the first time to observe motion of the positive charges in solid helium at temperatures below $0.1 \mathrm{~K}$. The current-voltage dependence could be described by a power law $I \sim U^{\alpha}$, with the value of the exponent $\alpha \gg 2$, much higher than what one would expect for the regime of space charge limited currents. (C) 2015 AIP Publishing LLC.
\end{abstract}

[http://dx.doi.org/10.1063/1.4927049]

\section{Introduction}

Properties of injected charges in solid helium were investigated in detail at temperatures $T>0.5 \mathrm{~K}$ by different authors (see the review report by $\mathrm{Dahm}^{1}$ for example). However, the nature of the local maximum on the temperature dependence of mobility of the positive charges observed at $T \sim 0.8 \mathrm{~K}$ in hcp ${ }^{4} \mathrm{He}$ crystals grown at pressures of 30-40 bar, as well as the strong nonlinear dependence of the charge velocity on the voltage applied, ${ }^{2}$ is not understood so far. The previous measurements were limited by the temperature $T>0.5 \mathrm{~K}$ due to relatively high heat emission, $10^{-4} \mathrm{~W} / \mathrm{cm}^{2}$, by radioactive sources of ionization used in those experiments.

The purpose of the present research was the preparation of a plane source of charges with low heat emission (less than $10^{-6} \mathrm{~W} / \mathrm{cm}^{2}$ ) suitable for studies of motion of injected charges in solid helium at low temperatures down to $0.1 \mathrm{~K}$.

Several types of sources which are used for the charge injection into bulk condensed helium are known. One of the most accepted now is field-emission sources, using sharp metal tips fabricated by etching a tungsten wire. ${ }^{3}$ The value of voltage $U$ applied to the tip is usually several hundred volts, the current $I$ being up to $10^{-10}$ A. Though the heat flux density from the tip $I U / S$, where $S$ is the area of the tip, is relatively large, the usage of a metal tip as a source of charges in liquid helium enables one to work with shortpulse techniques to avoid overheating the surrounding superfluid liquid. Unfortunately local overheating of a solid sample around the tip even for a short time might result in generation of a number of defects in the crystal that restricts usage of a single metal tip as a source of injected charges in solid helium.

Keeping in mind all of this we attempted to prepare a plane source of charges based on many carbon nanotubes on a metal substrate. The diameter of free end of the nanotube is of the order of $1 \mathrm{~nm}$, comparable with the radius of curvature of metal tips. ${ }^{4}$ The carbon tubes are stable in air. A successful example of the application of nanotubes in liquid helium is described in work ${ }^{5}$ where the authors had observed emission of charges from a metal rod coated by nanotubes upon the application of voltage $U=100 \mathrm{~V}$ for negative charges and $U=400 \mathrm{~V}$ for generation of positive charges into bulk liquid.

\section{Preparation of the nanotube sources}

The procedure of fabrication of the plane charge sources, based on the field-emission from carbon nano-tubes, is described in details in our paper. ${ }^{6}$

Two methods of fabrication appeared to be the most successful. In the first method, carbon nanotubes produced by the arc technique were deposited onto copper substrates $10 \mathrm{~mm}$ in diameter. ${ }^{7}$ The device for producing nanotube arrays on metal substrates is described in Ref. 8. 3-5 polished metal substrates were placed on a flat graphite cathode and fixed with graphite screws, so that the distance from the cathode center to the substrate center was $12-20 \mathrm{~mm}$. 


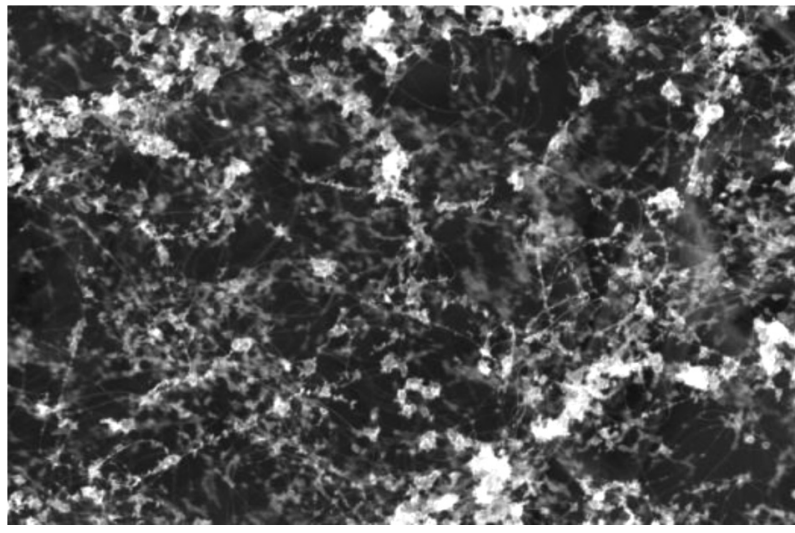

FIG. 1. SEM photograph of copper substrate surface covered with carbon structures.

Deposition was performed in an argon atmosphere, the time for preparation varying between 5 and $30 \mathrm{~s}$.

Figure 1 shows the copper substrate surface covered with the carbon products deposited from the gas discharge. The photographs made with a scanning electron microscope reveal clearly defined, long agglomerated nanotubes. Larger carbon structures are seen in the intersecting points of the nanotubes.

In the second method the substrate surface was precovered with carbon black and then the nanotubes were uniformly scattered over the surface. The mixture obtained was rubbed into the porous substrate surface with the polished end of a steel cylinder to achieve a high surface finishing. The access carbon black and nanotubes were removed from the substrate surface in the course of rubbing. Experiment showed that the use of carbon black improves the mechanical contact of the nanotubes with the metal surface. The distribution of nanotubes over the surface is close to that shown in Fig. 1.

\section{Experimental techniques}

The efficiency of a source was tested in an experimental cell filled with superfluid helium. The source of charges was placed at the bottom plate of a plane diode. The upper plate served as a collector. The source-collector distance was $0.5 \mathrm{~mm}$. An important characteristic of the source is the threshold value of voltage $U$ above which the current through the cell in $\mathrm{He}$ II reaches an appreciable value (exceeds $10^{-13} \mathrm{~A}$ in our tests). The voltage from a Stanford Research Systems, Model PS350/5000V-25W dc voltage source was applied to the cell through $108 \Omega$ resistance. The collector current was measured with a Stanford Research Systems, Model SR 570 dc current amplifier.

Figure 2 presents the voltage-current characteristic of the source prepared by the first method. The source was placed into superfluid helium cooled down to $1.3 \mathrm{~K}$. Negative voltage is applied to the source so that negative charges (electrons, localized in vacuum bubbles of $\sim 1.7 \mathrm{~nm}$ radius) move through the liquid from the source to the collector (curve 1). Positive charges (snowballs of $1 \mathrm{~nm}$ diameter, consisted of an ionized helium ion and a layer of solidified helium around it) are created in He II when positive voltage is applied to the source. ${ }^{9}$

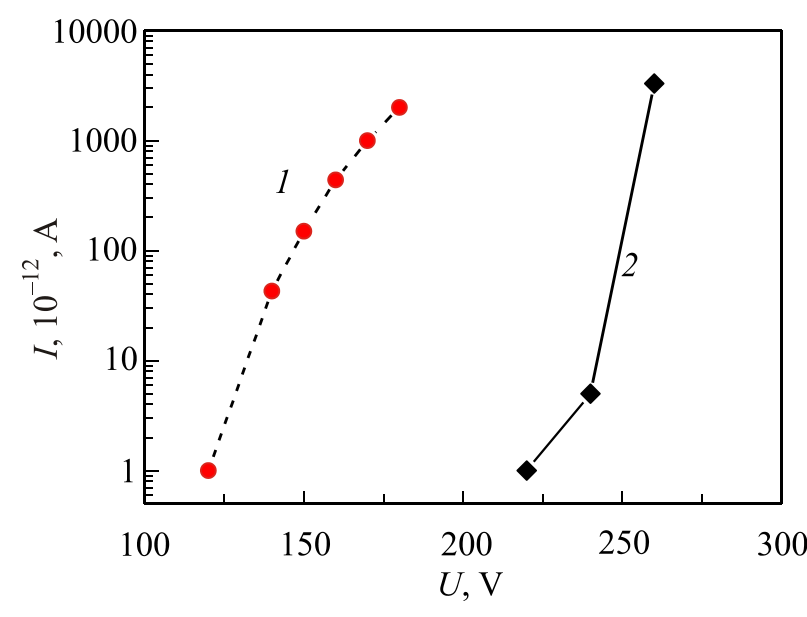

FIG. 2. Voltage-current dependences of the diode in superfluid He II at 1.3 K. Negative charges (curve 1), positive charges (curve 2).

As seen from Fig. 2, a noticeable negative current can be detected at a voltage $U=-120 \mathrm{~V}$. After changing the polarity of the voltage applied to the source from negative to positive, we have detected the current of positive charges (ions) at voltages $U \geq 220 \mathrm{~V}$ (curve 2).

In experiments with charges in liquid and solid helium at low temperatures, it is of importance to avoid any local overheating of the liquid surrounding the nanotubes, i.e., to achieve more or less uniform distribution of the centers of emission. To check that charges are not emitted by just a few nanotubes, but from different points on the source surface, the collector was divided in two halves, and the currentvoltage dependences of both parts were measured in superfluid He II. Figure 3 shows the voltage dependences of the positive current from both parts of the collector (squares and circles) and their sum (triangles).

One can see that the field dependences of both halves of the source are nearly identical, that implies a uniform distribution of the emission centers over the source surface. Yet, sometimes the field characteristics of the two parts differed significantly. This means that each source should be tested for the uniformity of the distribution of the electron emission centers over the source surface.

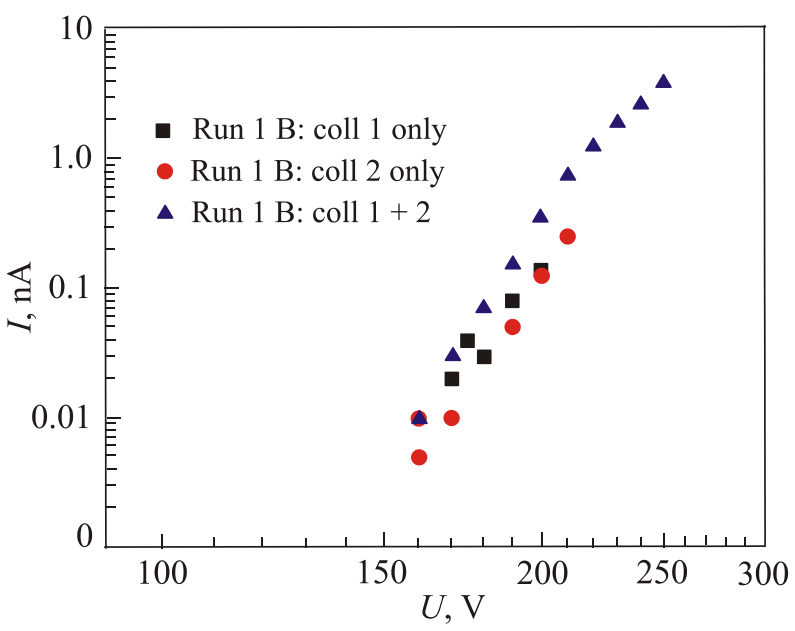

FIG. 3. The current of positive charges in superfluid He II as a function of voltage applied to the source at $1.3 \mathrm{~K}$ : the squares denote the first half, the circles the second half, and the triangles-the sum of the currents from both halves. 


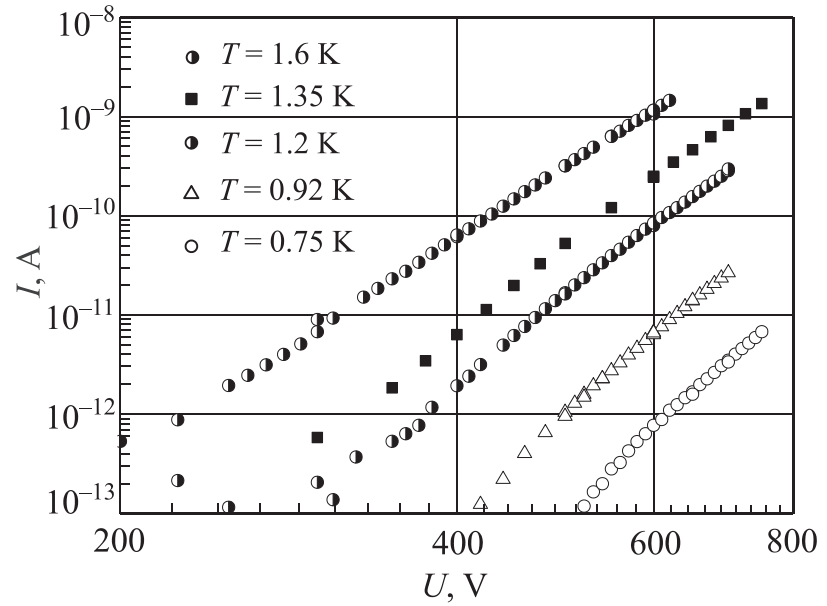

FIG. 4. Field dependences of the current of negative charges in the solid helium at different temperatures from 1.6 to $0.75 \mathrm{~K}$.

\section{Experiments with solid helium}

Samples of solid ${ }^{4} \mathrm{He}$ were grown by the blocked capillary method. Prior to sample growth the liquid in the cell was pressurized to 71 bar at $T=3 \mathrm{~K}$. Then the cell was slowly cooled down to $0.23 \mathrm{~K}$. The solid sample was growing in a blocked cell, so the pressure in the cell dropped gradually in accordance with the $P-T$ diagram. The residual pressure in the cell was about $40 \mathrm{~atm}$. Before the current measurements the sample was annealed at temperature $T=1.6 \mathrm{~K}$ for $2 \mathrm{~h}$.

The voltage dependences of the negative charge current in the sample of solid ${ }^{4} \mathrm{He}$ at different temperatures are shown in Fig. 4 in the log-log scale. Note that in a wide temperature range all the experimental curves (points) could be described by a power function of voltage $I \sim U^{\alpha}$ and the exponent $\alpha$ is increasing from $\sim 6$ to 10 with decreasing temperature from 1.6 to $0.75 \mathrm{~K}$.

The field dependences of the positive charge currents in solid helium at several temperatures between 1.43 and $0.075 \mathrm{~K}$ are shown in Fig. 5. It should be noted that we could observe motion of the positive charges in solid helium below $0.1 \mathrm{~K}$ for the first time. These observations open new opportunities for further investigations of the mechanisms of diffusion of charged point defects in quantum crystals. The field dependence of the positive current below $1 \mathrm{~K}$ can be

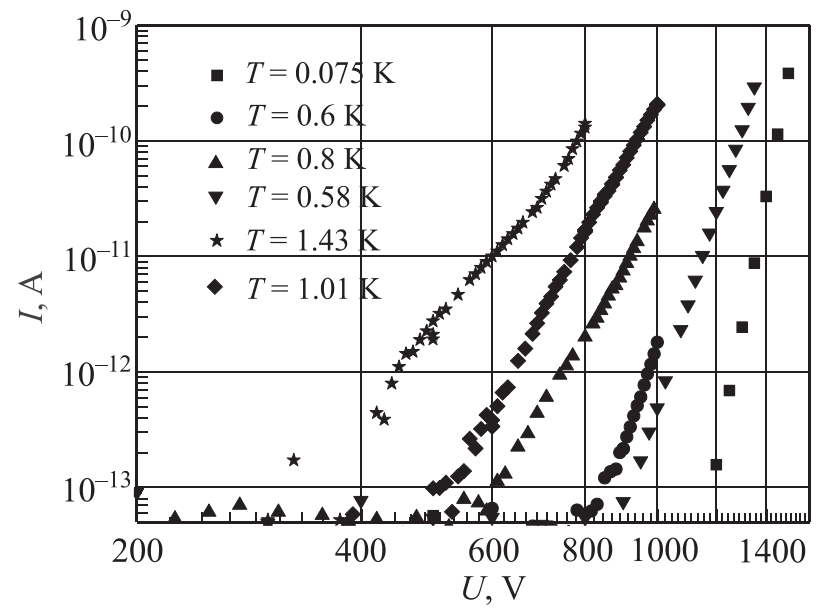

FIG. 5. Field dependences of positive charge current in a sample of solid helium at temperatures between 1.43 and $0.075 \mathrm{~K}$. described by the power law function of $I \sim U^{\alpha}$, and the values of the exponent $\alpha$ at with lowering the temperature from $1.01 \mathrm{~K}$, to 0.58 and then to $0.075 \mathrm{~K}$ increase from $\alpha=12$ to 21 , and then to 37 .

From the comparison of the results presented in Figs. 4 and 5, it follows that the field dependences of the current of positive charges are steeper than those for negative charges. However, in both cases the experiment values of the exponent $\alpha$ are much greater than the value $\alpha=2$ predicted by the theory for the regime of space charge limited currents.

The observed sharp field dependence can be explained by the following factors.

(1) The heterogeneity of the surface of the nanotubes based source of charges. The flat surface of metal substrate is covered with a film of strongly entangled and disordered carbon nanotubes. The total current emitted from the surface is determined by the voltage applied and by the quantity and the structure of the protruding parts of the tubes. ${ }^{4}$ It is clear that the field dependence of the current emitted from the source should be strongly nonlinear.

(2) The presence of a large number of microscopic defects in a sample of solid helium prepared by the blocked capillary method. These defects can serve as traps for the charged point defects moving through the sample. ${ }^{10}$ The capture of the mobile charges by the defects of different nature (dislocations, grain boundaries) in the sample at small electric fields and its release from the shallow traps with increasing the voltage applied should strongly affect the field and temperature dependences of the current of injected charges in solid helium.

(3) The strong voltage dependence of charge drift velocity in strong electric fields higher than $104 \mathrm{~V} / \mathrm{cm}$. If we assume that the main mechanism of the point charged defect motion is thermally activated diffusion over the potential barrier in the presence of an external electric field, than in strong electric fields the charge velocity should be a nonlinear function of voltage applied, ${ }^{1,11}$ and the current through the sample should be proportional to hyperbolic sine $\mathrm{I} \sim \exp (-\Delta / \mathrm{T}) \sinh ($ Eea/kT), where $\Delta$ is the height of the barrier, $\mathrm{E}$ is the electric field, and a is a lattice constant.

The temperature dependences of current in strongly disordered samples have a distinct activation character. The value of activation energy of charge motion calculated from the temperature dependences of currents are close to $10 \mathrm{~K}$.

However, as it was mentioned above in the case of positive charges the current is recorded at a temperature well below $0.1 \mathrm{~K}$. Taking into account the value of the current at $0.6 \mathrm{~K}$ and $U=1300 \mathrm{~V}$, in order to get the observed magnitude of current at $75 \mathrm{mK}$ one should assume that the activation energy is close to $0.3 \mathrm{~K}$ at low temperatures. Unfortunately, the existing experimental data are insufficient to make an unequivocal conclusion that it is the activation energy that changed with decreasing temperature and not the mechanism of charges motion.

\section{Conclusion}

Methods of fabrication of cold charges based on carbon nanotubes have been developed for low temperature 
experiments. First investigations of the motion of charges in solid helium with the new charges sources were carried out in samples of solid helium. It was shown that the motion of positive charges can be observed at temperatures below $0.1 \mathrm{~K}$. To clarify the questions on the mechanisms of diffusion of injected charges in solid helium at low temperatures and in high electric fields further experimental studies are needed.

This work was partially supported by Grant RFRB No. 1202-00581.

a)Email: levch@issp.ac.ru

${ }^{1}$ S. C. Lau and A. I. Dahm, J. Low Temp. Phys. 112, 47 (1998).

${ }^{2}$ L. P. Mezhov-Deglin, V. B. Efimov, and A. I. Golov, Fiz. Nizk. Temp. 10, 99 (1984) [Sov. J. Low Temp. Phys. 10, 53 (1984)].
${ }^{3}$ A. Golov and H. Ishimoto, J. Low Temp. Phys. 113, 957 (1998).

${ }^{4}$ A. V. Eletskii, Phys. Usp. 53, 863 (2010).

${ }^{5}$ K. Kawasaki, K. Tsukagoshi, and K. Kono, J. Low Temp. Phys. 138, 899 (2005).

${ }^{6}$ D. N. Borisenko, P. M. Walmsley, A. I. Golov, N. N. Kolesnikov, Yu. V. Kotov, A. A. Levchenko, and M. Fear, Instrum. Exp. Tech. 57, 755 (2014).

${ }^{7}$ D. N. Borisenko, N. N. Kolesnikov, M. P. Kulakov, and V. V. Kveder, Int. J. Nanoscience 1(3-4), 235 (2002).

${ }^{8}$ N. N. Kolesnikov, D. N. Borisenko, and A. A. Levchenko, Russian patent 2471706 (2013).

${ }^{9}$ V. B. Shikin, Phys. Usp. 20, 226 (1977).

${ }^{10}$ M. Lampert and P. Mark, Injection Currents in Solids (Mir, Moscow, 1973).

${ }^{11}$ V. B. Efimov and L. P. Mezhov-Deglin, JETP Lett. 33, 521 (1981).

This article was published in English in the original Russian journal. Reproduced here with stylistic changes by AIP Publishing. 